\title{
Score for predicting overall survival in pancreatic adenocarcinoma patients with positive lymph nodes after surgery: a novel nomogram-based risk assessment
}

\author{
Liang Jin, Yiping Zou, Shiye Ruan, Hongwei Han, Yuanpeng Zhang, Zhihong Chen, Haosheng Jin, \\ Ning Shi \\ Department of General Surgery, Guangdong Provincial People’s Hospital, Guangdong Academy of Medical Sciences, Guangzhou, China \\ Contributions: (I) Conception and design: L Jin; (II) Administrative support: N Shi, H Jin; (III) Provision of study materials or patients: L Jin, N \\ Shi; Y Zou; (IV) Collection and assembly of data: L Jin, H Han, Y Zhang, Z Chen; (V) Data analysis and interpretation: L Jin, Y Zou, S Ruan; (VI) \\ Manuscript writing: All authors; (VII) Final approval of manuscript: All authors. \\ Correspondence to: Prof. Haosheng Jin; Prof. Ning Shi. Department of General Surgery, Guangdong Provincial People’s Hospital, Guangdong \\ Academy of Medical Sciences, Guangzhou 510080, China. Email: kinghaos@126.com; shining_doc@163.com.
}

Backgrounda Pancreatic adenocarcinoma $(\mathrm{PaC})$ patients with positive lymph nodes (PLNs) have a dismal
prognosis and lack a specific prognostic stage. This study aimed to construct a nomogram for the prediction
of overall survival $(\mathrm{OS})$ in these patients.

Methods: A total of 1,340 patients screened from the Surveillance, Epidemiology, and End Results database were included and randomly divided at a ratio of 7:3 into a training set $(n=940)$ and an internal validation set $(n=400)$. Cox regression analyses were conducted to select independent predictors in the training set, and a nomogram was constructed. The model was verified in the internal validation set and in an external validation set, which comprised 64 patients from a Chinese institute.

Results: Six independent prognostic factors (age at diagnosis, tumor grade, lymph node ratio, $T$ stage, radiotherapy, and chemotherapy) were identified in $\mathrm{PaC}$ patients with PLNs and were entered into the nomogram. The final model had a higher C-index for predicting OS than the American Joint Committee on Cancer-8th edition staging system (training set: 0.658 vs. 0.546 ; internal validation set: 0.661 vs. 0.546; external validation set: 0.691 vs. 0.581 ). The 1-, 2-, and 3-year area under the receiver operating characteristic curve values indicated better discrimination power for the established nomogram with respect to the prediction of OS in the training, internal validation, and external validation sets than for the American Joint Committee on Cancer-8th edition staging system. Furthermore, the nomogram performed well in both calibration and decision curve analyses (DCA) of clinical applicability. OS in PaC patients with PLNs was significantly distinguished among the three risk groups stratified according to the nomogram score $(\mathrm{P}<0.001)$. Conclusions: The well-calibrated nomogram was determined to be extremely efficient in predicting survival, and defining a high-risk population based on the nomogram score among $\mathrm{PaC}$ patients with PLNs after surgery.

Keywords: Positive lymph nodes (PLNs); pancreatic adenocarcinoma (PaC); nomogram; overall survival (OS); C-index

Submitted Jul 07, 2020. Accepted for publication Dec 01, 2020.

doi: $10.21037 /$ gs-20-597

View this article at: http://dx.doi.org/10.21037/gs-20-597 


\section{Introduction}

Pancreatic adenocarcinoma $(\mathrm{PaC})$ represents one of the most lethal malignancies among humans. Surgical resection remains the mainstay therapy for $\mathrm{PaC}$; nonetheless, $<20 \%$ of patients are considered candidates for resection $(1,2)$, and the 5-year survival remains disappointing even if a curative resection is performed (3-5). It is likely that the implementation of a reasonable treatment strategy remains imperfect. Considering the high rate of postoperative local recurrence (6), most $\mathrm{PaC}$ patients will ultimately die from local progression.

The National Comprehensive Cancer Network (NCCN) guidelines clearly state that $\mathrm{PaC}$ patients with positive lymph nodes (PLN-PaCs) are specific individuals with a high recurrence risk (7). While lymph node (LN) status is a strong prognosticator for overall survival (OS) (8), insufficient LN examination may lead to the misclassification of $\mathrm{N} 1$ disease as $\mathrm{N} 0$ disease in some $\mathrm{PaC}$ patients (9). Therefore, PLN-PaCs with a high recurrence risk should be regarded as a population distinct from other $\mathrm{PaC}$ patients.

The American Joint Committee on Cancer (AJCC) staging system is currently the tool widely used by oncologists for predicting the prognosis of $\mathrm{PaC}$ patients (10). However, the AJCC staging system does not consider specific individuals, including the tumor differentiation grade (11), therapy method $(12,13)$, and number of regional LNs examined. In reality, various factors can influence the cancer course and prognosis of $\mathrm{PaC}$ patients, and refining and establishing a new model for specific individuals (i.e., PLN-PaCs) are thus important.

The use of a nomogram, a novel statistical prediction model, can accurately estimate individual survival and guide plans for follow-up by integrating multiple factors (14). Furthermore, several studies have indicated the favorable results of nomogram (15) and its utility in various cancers (16-19). To date, an effective nomogram for PLN-PaCs has never been developed. Hence, the present study aimed to explore critical prognostic factors and construct a novel nomogram for predicting the prognosis of PLN-PaCs based on the Surveillance, Epidemiology, and End Results (SEER) database, which contains data from a large population. Furthermore, the novel nomogram was validated using data from Guangdong Provincial People's Hospital in China.

We present the following article in accordance with the TRIPOD reporting checklist (available at http://dx.doi. org/10.21037/gs-20-597).

\section{Methods}

\section{Patient selection}

Data of PLN-PaCs were acquired from the SEER database using SEER*Stat software version 8.3.5 (www.seer.cancer. gov/seerstat). These patients were selected based on the 2nd and 3 rd editions of the International Classification of Diseases for Oncology (ICD-O-2/3), and "8140/3" was used as the ICD-O-3 diagnosis to identify $\mathrm{PaC}$.

Patients were included only if they were PLN-PaCs. The exclusion criteria were as follows: fewer than 11 regional LNs examined or unknown number of regional LNs; distant metastases; receiving other forms of treatment aside from surgery; survival time $\leq 1$ month; second primary cancer; and missing or incomplete data (Figure 1).

\section{Data processing}

A total of 1,340 eligible PLN-PaCs from the SEER database were finally included in our retrospective study based on the above mentioned inclusion and exclusion criteria. External validation data $(n=64)$ were collected from Guangdong Provincial People's Hospital in China. Data on pathological and clinical variables [e.g., age at diagnosis, sex, tumor size, tumor differentiation, tumor-node-metastasis (TNM) stage, chemotherapy, radiotherapy, LNs] as well as follow-up information were extracted from the Guangdong Provincial People's Hospital and SEER databases. LN ratio (LNR) was defined as the ratio of the number of metastasized LNs to the total number of resected LNs. Information on TNM-8th edition staging was gathered based on the 7 th edition of the AJCC staging system (2010+), and tumor size and number of PLNs were used to estimate the AJCC-8th edition stage.

Risk factors were extensively evaluated by conducting a univariate analysis of all included variables, and a multivariate analysis was adopted to select independent risk factors. A nomogram was constructed based on these independent risk factors and was validated in both training and external validation cohorts.

\section{Nomogram development and external validation}

For nomogram construction, PLN-PaCs $(n=1,340)$ from the SEER database [2010-2015] were randomly divided at a ratio of $7: 3$ into a training set $(n=940)$ and an internal validation set $(n=400)$. The training set was used to construct the nomogram, and the benefits of the novel model were evaluated by internal and external validation. The second 


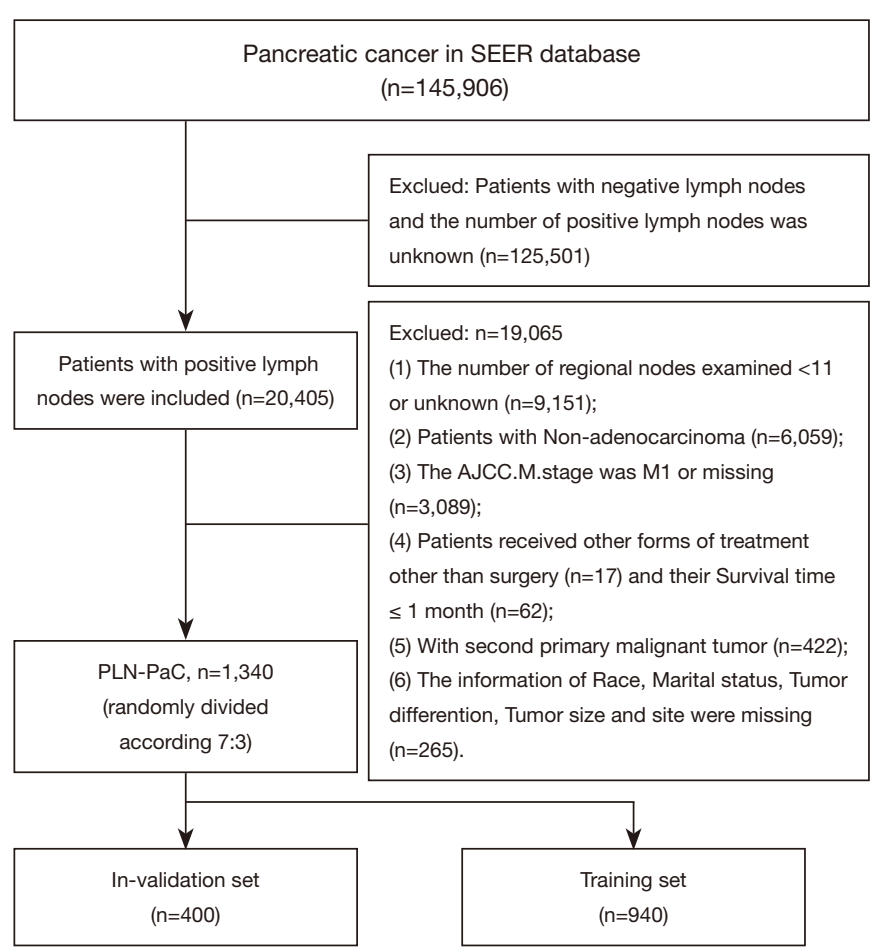

Figure 1 Flow diagram illustrating patient selection.

cohort consisted of patients from Guangdong Provincial People's Hospital $(n=64)$ and was used as an external validation cohort [2010-2019]. Furthermore, the predictive power of the established nomogram was compared with that of the 8th edition of the TNM staging system.

\section{Statistical analyses}

Baseline patient demographics and disease features were compared using the chi-square test or Fisher's exact test, as appropriate. Statistical analyses were performed using SPSS software version 25 (IBM Corp., Armonk, NY, USA). Cox multivariate regression and the nomogram were constructed using the rms package in $\mathrm{R}$ version 3.6.1 (http:// www.r-project.org/). For outcome-based optimization, the best cut-off points for age, LNR, tumor size, and nomogram score (nomo-score) were calculated using X-tile software (Yale School of Medicine, New Haven, CT, USA). $\mathrm{P}$ values were two-sided, and $\mathrm{P}$ values lower than 0.05 were considered statistically significant. The $\mathrm{C}$-index and area under the receiver operating characteristic (ROC) curve (AUC) were used to evaluate the discriminative ability of the nomogram (20). Calibration curves were utilized to examine the association between actual outcomes and

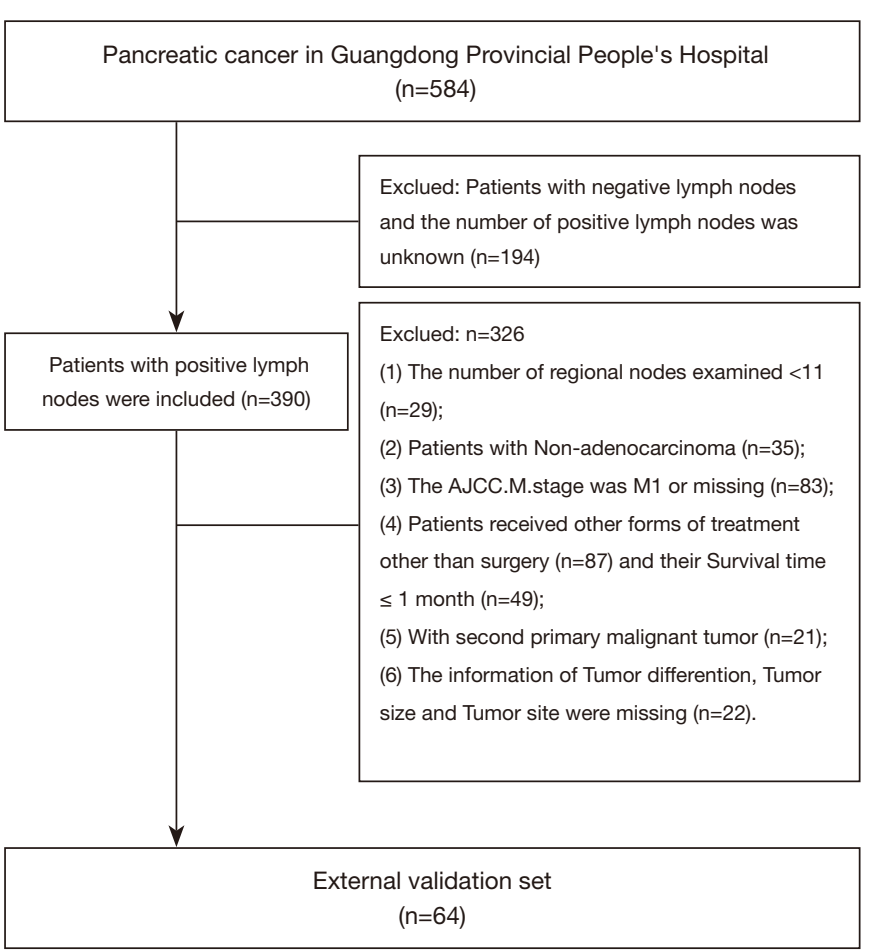

predicted probabilities (21). The clinical usefulness and benefits of the prediction model were estimated using decision curve analyses (DCA) (22).

\section{Ethical statement}

The study was conducted in accordance with the Declaration of Helsinki (as revised in 2013).

\section{Results}

\section{Characteristics of PLN-PaCs}

The PLN-PaCs $(n=1,340)$ from the SEER database were randomly divided at a ratio of 7:3 into a training set $(\mathrm{n}=940)$ and an internal validation set $(\mathrm{n}=400)$. For these PLN-PaCs from the SEER database, the median survival time (MST) was 20 months, whereas the 1-, 2-, and 3-year OS rates were $70.5 \%, 40.3 \%$, and $25.1 \%$, respectively.

A total of 64 PLN-PaCs from Guangdong Provincial People's Hospital were allocated to the external validation set. The clinicopathological characteristics, including age, sex, radiotherapy, chemotherapy, and TNM stage, are summarized in Table 1. The MST was 23.8 months, whereas 
the 1-, 2-, and 3-year OS rates were $77.3 \%, 47.5 \%$, and $30.7 \%$, respectively. Additionally, for these 64 PLN-PaCs, the median disease-free survival (DFS) was 10.3 months, whereas the 1-, 2-, and 3-year DFS rates were $44.3 \%$, $26.8 \%$, and $22.4 \%$, respectively.

\section{Screening of prognostic factors for PLN-PaCs}

Multivariate analysis was performed to identify independent prognostic factors in the training set. In order to avoid the influence of tumor size on AJCC T stage and of AJCC $\mathrm{N}$ stage on LNR, tumor size and AJCC N stage were not included in the multivariate analysis. Univariate and multivariate analyses revealed that age, tumor differentiation grade, LNR, AJCC T stage, radiotherapy, and chemotherapy were independent prognostic factors for OS (Table 2).

\section{Nomogram construction}

Based on the above mentioned results of multivariate analysis from the training set, we integrated these independent prognostic factors to establish a satisfactory nomogram for OS prediction in PLN-PaCs (Figure 2). The detailed scores of all variables in the nomogram are presented in Table S1. By summing the detailed score of each variable, we could obtain a nomo-score to predict the possibility of 1-, 2-, and 3-year OS, with a higher nomoscore indicating worse prognosis.

\section{Validation and calibration of the nomogram}

With respect to the 1-, 2-, and 3-year OS, the AUC values indicated better discriminative ability for this model than for the traditional AJCC-8th edition staging system in the training set (1-year: $0.717 v s$. 0.565, 2-year: 0.675 vs. 0.549 , 3 -year: $0.678 v$ s. 0.562 ; Figure $3 A, B)$, internal validation set (1-year: 0.721 vs. 0.577, 2-year: 0.719 vs. $0.541,3$-year: 0.700 vs. 0.544; Figure 3C,D), and external validation set (1-year: 0.898 vs. 0.626, 2-year: 0.748 vs. 0.677, 3-year: 0.775 vs. 0.629; Figure 3E,F). Furthermore, the nomogram had a higher C-index than the AJCC staging system only in the training set $(0.658 v s$. 0.546$)$ and internal validation set (0.661 vs. 0.546). The C-index of the nomogram was also higher than that of the AJCC staging system in the external validation set (0.691 vs. 0.581).

The calibration curves indicated an acceptable agreement between the actual and predicted outcomes for the 1-, 2-, and 3-year probabilities of OS in the training set (Figure 3G), internal validation set (Figure $3 H$ ), and external validation set (Figure 3I). Importantly, the DCA of clinical applicability revealed that the established nomogram had greater net benefits across a range of death risk than the AJCC-8th edition staging system in the training cohort (Figure 4).

\section{Performance of the nomogram in stratifying patient risk}

The training set was stratified into three risk groups according to the nomo-score, with the cut-off value determined by $\mathrm{X}$-tile software-namely, low-risk group: $0 \leq$ nomo-score $\leq 174$; middle-risk group: $177<$ nomo-score $\leq 262$; and high-risk group: nomo-score $>264$. Kaplan-Meier survival curves for OS in the three risk groups according to the nomo-score were plotted (Figure 5), which showed an obvious grading ability based on the new risk group model $(\mathrm{P}<0.001)$.

\section{Discussion}

$\mathrm{PaC}$ is a heterogeneous disease that may lead to different prognoses in different patients, even after radical resection (23). PLN-PaCs are a population distinct from $\mathrm{PaC}$ patients owing to their low resection rates and high recurrence risk, making prognostic studies on PLN-PaCs who had undergone surgical resection difficult. Several previous studies have attempted to predict the prognosis of $\mathrm{PaC}$ patients; nevertheless, most of these studies lack representativeness because they were based on a broad pancreatic cancer population and had no restrictions on pathological type, specific individuals, number of regional LNs examined, or surgery status $(24,25)$. Therefore, developing and validating an effective nomogram with better applicability for PLN-PaCs is still necessary. To the best of our knowledge, the present study is the first largesample study to identify critical prognostic factors for PLNPaCs. Importantly, we established an easy-to-use nomogram based on clinicopathological and personalized characteristics to predict the prognosis of specific $\mathrm{PaC}$ individuals at a personal level, which is consistent with the concept of individualized treatment for cancer patients.

In the present study, we established a novel nomogram for PLN-PaCs by integrating age, tumor differentiation grade, AJCC T stage, LNR, chemotherapy, and radiotherapy. In the clinic, the 1-, 2-, and 3-year OS rates can be easily predicted using this nomogram, as it only includes six parameters, all of which are easy to acquire. Importantly, our nomogram exhibited higher predictive power than the AJCC-8th edition staging system in both the training and validation sets, which 
Table 1 Demographics and clinical characteristics of eligible patients with PLN-PaC

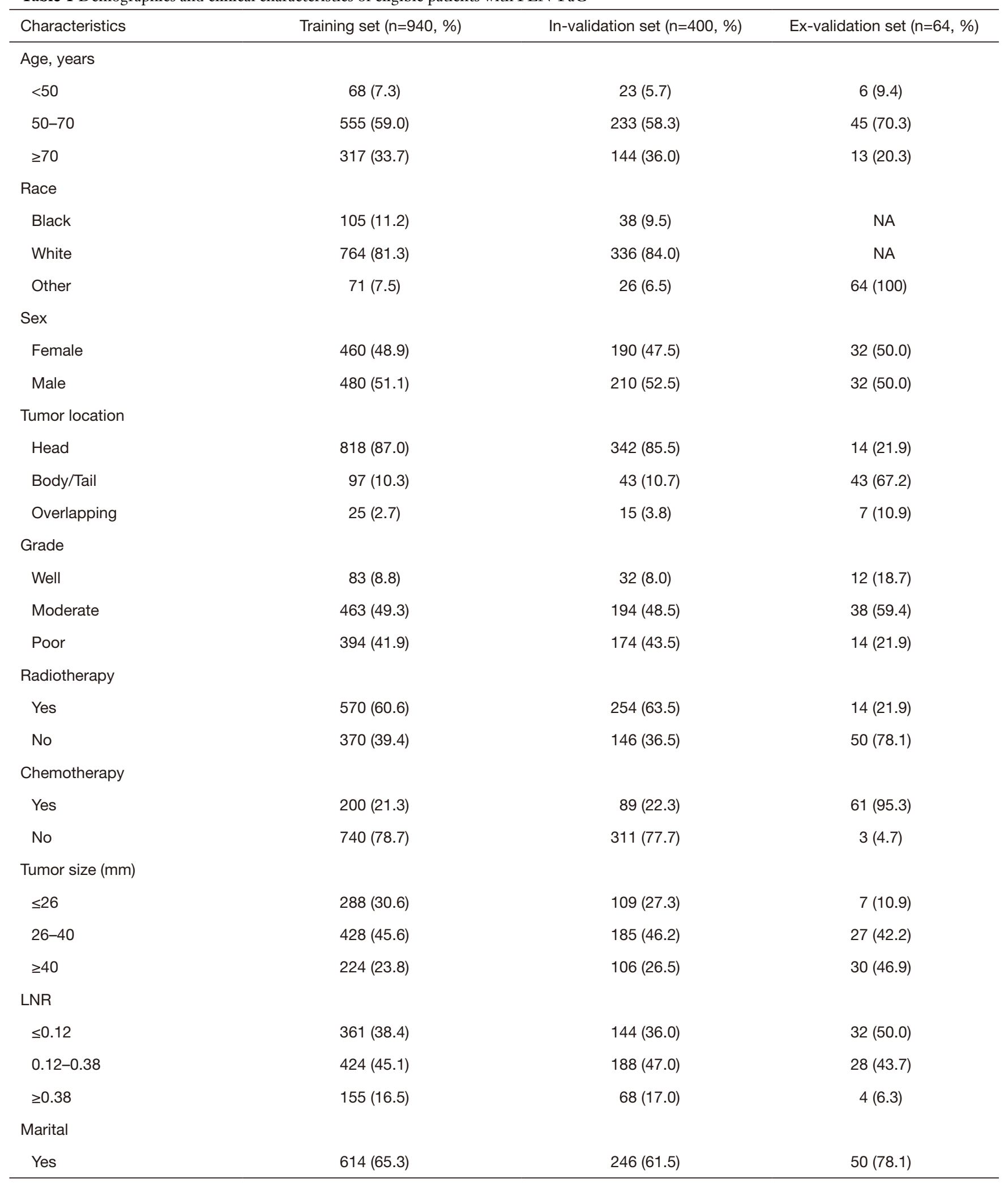

Table 1 (continued) 
Table 1 (continued)

\begin{tabular}{|c|c|c|c|}
\hline Characteristics & Training set $(n=940, \%)$ & In-validation set $(n=400, \%)$ & Ex-validation set $(n=64, \%)$ \\
\hline \multicolumn{4}{|l|}{ AJCC 8th stage } \\
\hline IIB & $482(51.3)$ & 198 (49.5) & $45(70.3)$ \\
\hline III & $458(48.7)$ & $202(50.5)$ & $19(29.7)$ \\
\hline $\mathrm{T} 1$ & $103(11.0)$ & $35(8.7)$ & $6(9.4)$ \\
\hline $\mathrm{T} 2$ & $580(61.7)$ & $251(62.7)$ & $24(37.5)$ \\
\hline T3 & $217(23.0)$ & 93 (23.3) & $20(31.2)$ \\
\hline $\mathrm{T} 4$ & $40(4.3)$ & $21(5.3)$ & $14(21.9)$ \\
\hline N2 & $442(47.0)$ & $191(47.7)$ & 8 (12.5) \\
\hline
\end{tabular}

PLN-PaC, pancreatic adenocarcinoma with positive lymph nodes; in-validation set, internal validation set; ex-validation set, external validation set; AJCC, American Joint Committee for Cancer; LNR, lymph nodes ratio.

Table 2 Univariate and multivariate cox regression analysis based on all variables for overall survival (training cohort)

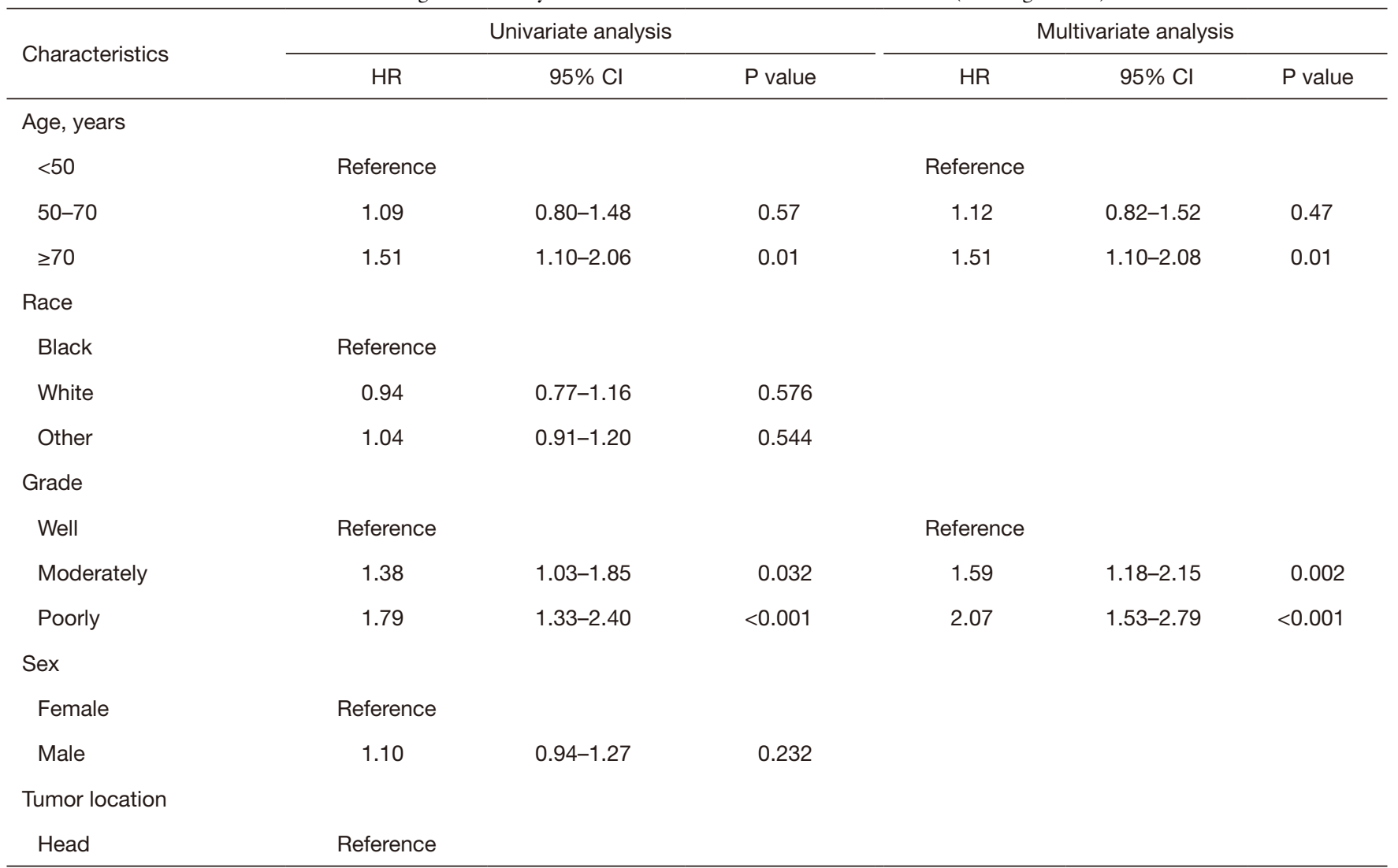

Table 2 (continued) 
Table 2 (continued)

\begin{tabular}{|c|c|c|c|c|c|c|}
\hline Characteristics & \multicolumn{3}{|c|}{ Univariate analysis } & \multicolumn{3}{|c|}{ Multivariate analysis } \\
\hline Body/tail & 0.89 & $0.68-1.15$ & 0.366 & & & \\
\hline Overlapping & 1.90 & $1.24-2.92$ & 0.003 & & & \\
\hline \multicolumn{7}{|l|}{ Radiotherapy } \\
\hline Yes & 0.67 & $0.57-0.78$ & $<0.001$ & 0.83 & $0.70-0.99$ & 0.03 \\
\hline \multicolumn{7}{|l|}{ Chemotherapy } \\
\hline No & Reference & & & Reference & & \\
\hline Yes & 0.48 & $0.40-0.57$ & $<0.001$ & 0.49 & $0.40-0.59$ & $<0.001$ \\
\hline \multicolumn{7}{|l|}{ LNR } \\
\hline $0.12-0.38$ & 1.29 & $1.09-1.52$ & 0.002 & 1.30 & $1.09-1.53$ & 0.002 \\
\hline$\geq 0.38$ & 1.85 & $1.50-2.29$ & $<0.001$ & 1.88 & $1.51-2.34$ & $<0.001$ \\
\hline \multicolumn{7}{|l|}{ Marital } \\
\hline No & Reference & & & & & \\
\hline Yes & 1.14 & $0.97-1.33$ & 0.11 & & & \\
\hline \multicolumn{7}{|l|}{ AJCC T stage } \\
\hline $\mathrm{T} 1$ & Reference & & & Reference & & \\
\hline $\mathrm{T} 2$ & 1.40 & $1.08-1.81$ & 0.009 & 1.35 & $1.04-1.75$ & 0.02 \\
\hline Total-PC & 1.14 & $0.86-1.51$ & 0.362 & & & \\
\hline \multicolumn{7}{|l|}{ Size $(\mathrm{mm})$} \\
\hline$\leq 26$ & Reference & & & & & \\
\hline $26-40$ & 1.36 & $1.14-1.62$ & $<0.001$ & NA & NA & NA \\
\hline$\geq 40$ & 1.61 & $1.32-1.97$ & $<0.001$ & NA & NA & NA \\
\hline \multicolumn{7}{|l|}{ AJCC N stage } \\
\hline $\mathrm{N} 1$ & Reference & & & & & \\
\hline N2 & 1.34 & $1.16-1.55$ & $<0.001$ & NA & NA & NA \\
\hline \multicolumn{7}{|l|}{ AJCC 8th stage } \\
\hline IIB & Reference & & & & & \\
\hline III & 1.36 & $1.18-1.58$ & $<0.001$ & NA & NA & NA \\
\hline
\end{tabular}

Partial-PC, partial pancreatectomy; total-PC, total pancreatectomy; AJCC, American Joint Committee for Cancer; LNR, lymph nodes ratio. 


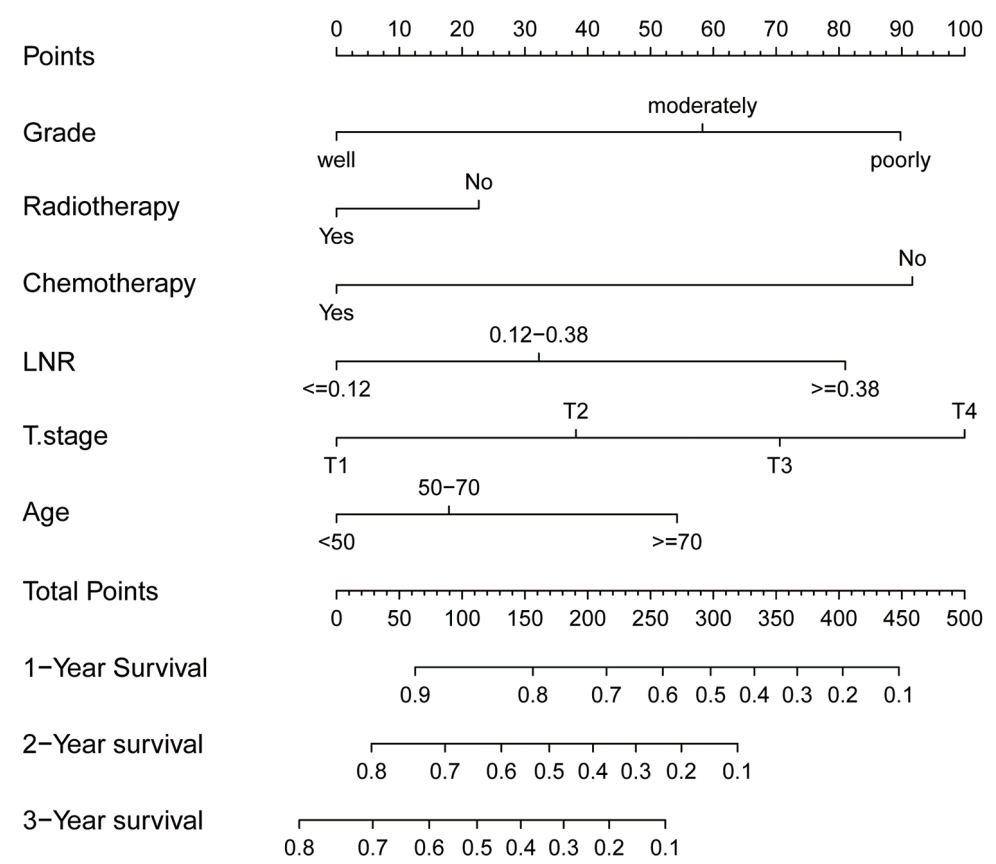

Figure 2 Nomogram for the prediction of overall survival in pancreatic adenocarcinoma patients with positive lymph nodes after surgical resection.

was confirmed by the higher $\mathrm{C}$-indexes and AUC values. The most likely reason for this is that the AJCC staging system takes the tumor size, number of PLNs, and metastasis into consideration; however, the differentiation grade and number of regional LNs examined are also independent critical factors for survival (26-28).

Indubitably, higher tumor differentiation grade and AJCC T stage are strongly associated with worse survival, as shown in our study and other previous investigations $(25,29)$. Unsurprisingly, $\mathrm{LN}$ status is an important predictor of survival. Recommendations for the number of LNs that should be examined range from 11 to 20 (30-35) in the case of pancreatectomy, and variable LNR categories are discussed in various studies (34-36). Additionally, several studies have reported that LNR may be a better prognostic indicator than the total number of positive nodes in node-positive patients $(30,37)$. Consequently, a sufficient number of regional LNs examined and accurate LNR enable accurate staging of patients and diminish the disparities in survival outcomes. To avoid understaging, we selected PLN-PaCs who underwent pancreatectomy and had at least 11 removed and examined LNs in our study. Our results indicated worse survival for PLN-PaCs who underwent pancreaticoduodenectomy and had $\geq 11 \mathrm{LNs}$ examined than for those who had $<11 \mathrm{LNs}$ evaluated [MST: 14 vs. 20 months; hazard ratio (HR), 0.7; 95\% confidence interval (CI), 0.61-0.80; $\mathrm{P}<0.0001]$. Moreover, our analysis showed that a greater LNR resulted in worse survival (HR, 1.88; 95\% CI, 1.51-2.34; $\mathrm{P}<0.001$ ).

Currently, adjuvant multi-agent chemotherapy followed by surgical resection is strongly recommended for resected PaCs (38). Correspondingly, our study also showed that chemotherapy was a protective factor, which was consistent with the findings of a clinical randomized controlled trial $(39,40)$.

Nevertheless, whether the administration of adjuvant radiotherapy to patients with resected $\mathrm{PaC}$ has a different effect on prognosis remains controversial. Some investigators argued that adjuvant radiotherapy did not significantly improve the $\mathrm{OS}$ of $\mathrm{PaC}$ patients, which was proven in our other studies (data are being published). However, we determined in our current study that radiotherapy was a critical factor that improved survival in PLN-PaCs. Based on the above mentioned results, the reasons for this may be that previous studies mixed up different histological types and recurrence risk groups in analyzing survival and did not identify specific individuals. Our results revealed that adjuvant radiotherapy did not improve survival in $\mathrm{PaC}$ patients with negative LNs (HR, 0.889; 95\% CI, 0.788-1.004; 
A

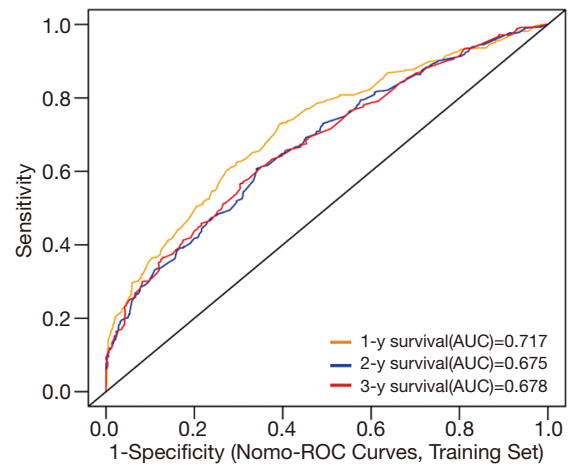

D

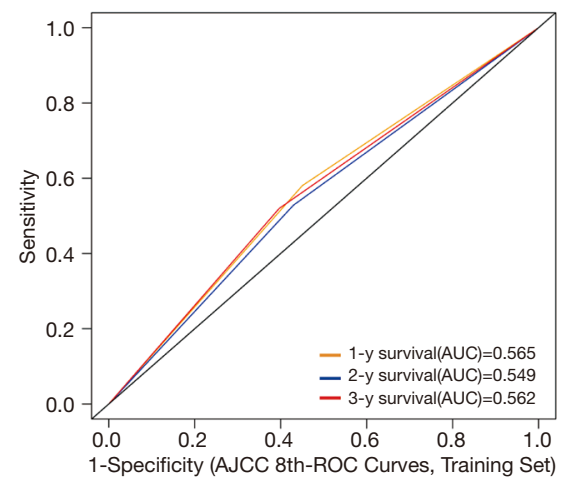

G

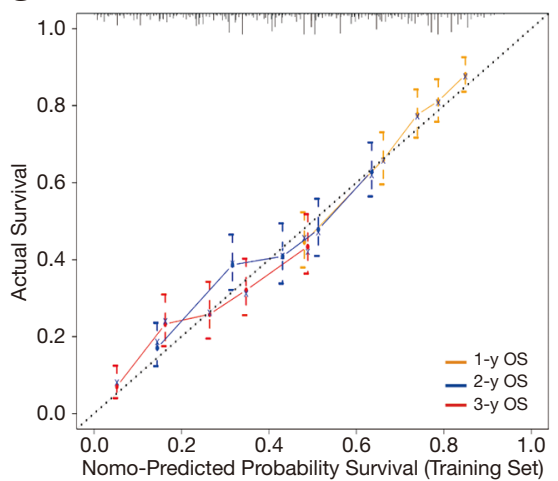

B

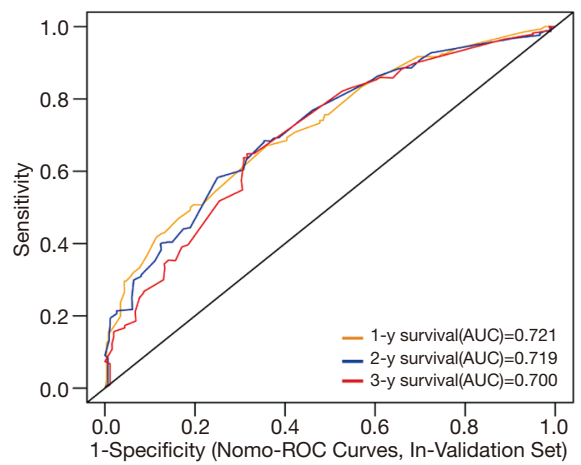

E

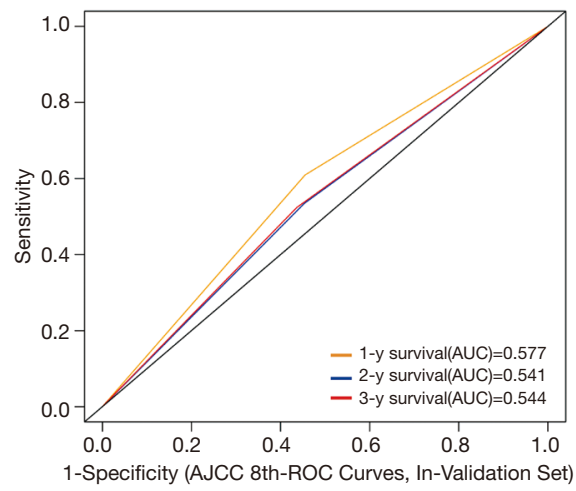

$\mathrm{H}$

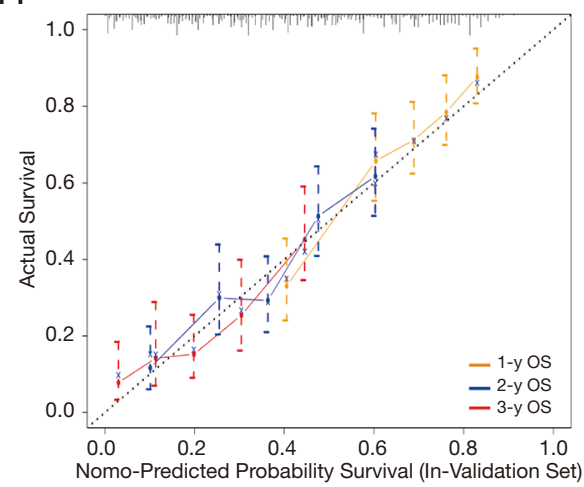

C

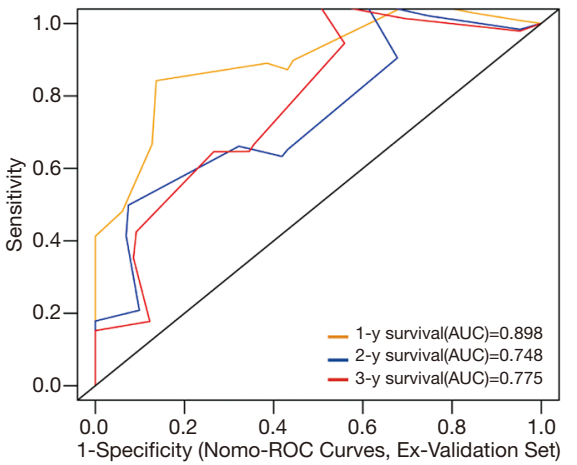

$F$

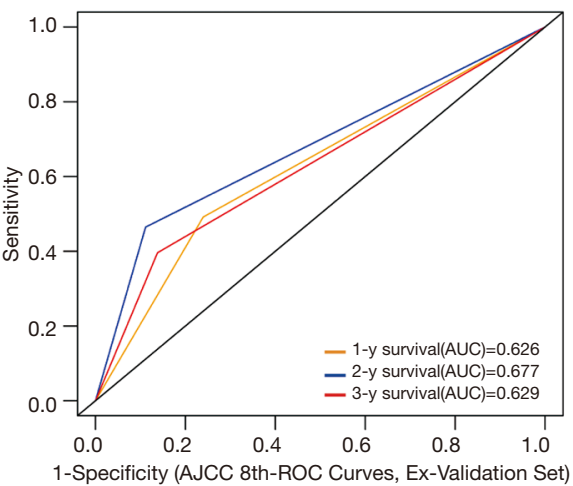

I

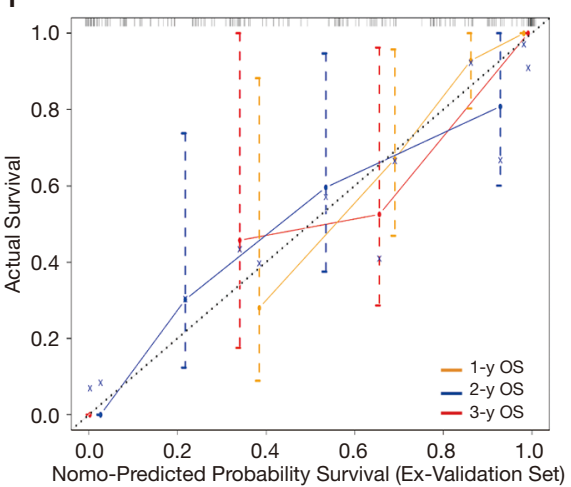

Figure 3 (A,C,E) Receiver operating characteristic (ROC) curves for the prediction of 1-, 2-, and 3-year overall survival (OS) based on the nomogram in the training, internal validation, and external validation sets. (B,D,F) ROC curves for the prediction of 1-, 2-, and 3-year OS based on the AJCC-8th edition staging system in the training, internal validation, and external validation sets. (G-I) Calibration plot for 1-, 2-, and 3 -year OS prediction based on the nomogram in the training, internal validation, and external validation sets.

$\mathrm{P}=0.06)$ but was a protective critical factor in PLN-PaCs (HR, 0.830; 95\% CI, 0.700-0.990; $\mathrm{P}=0.03)$. The NCCN guidelines point out that the role of adjuvant radiotherapy is still being evaluated in clinical studies; nonetheless, these guidelines also suggest that PLN-PaCs may receive radiotherapy after resection, which is consistent with our results. $\mathrm{As} \mathrm{PaC}$ is a heterogeneous disease that may lead to different prognoses in different patients even after radical resection, we should therefore treat specific $\mathrm{PaC}$ patients individually rather than generally.

The novel nomogram described in this study can be used to define a high-risk population with a total nomo-score of 
A

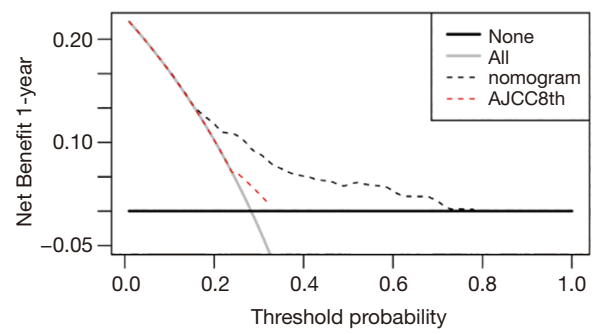

B

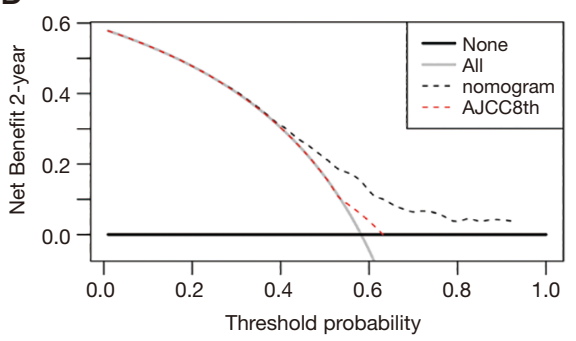

C

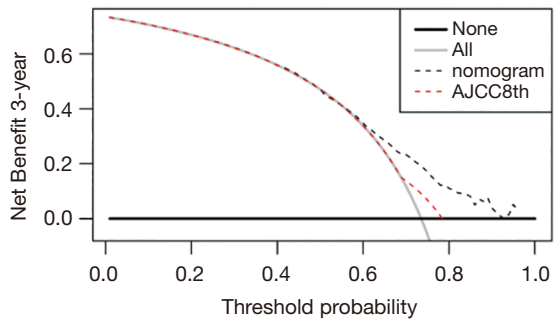

Figure 4 Decision curve analyses (DCA) of the nomogram and the American Joint Committee on Cancer (AJCC)-8th edition staging system in the training set. DCA of (A) 1-year risk, (B) 2-year risk, and (C) 3-year risk.

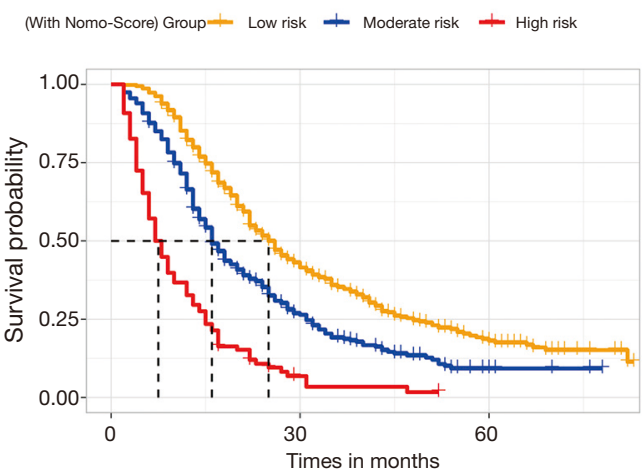

Figure 5 Kaplan-Meier curves for patients' overall survival (OS) among the low-risk, moderate-risk, and high-risk groups stratified according to the nomo-score in the training set.

>264 among PLN-PaCs. As shown in Figure 5, the KaplanMeier survival curves for OS indicate an obvious grading ability according to the new risk group model. Additionally, this effective nomogram was established for the first time using a Western database and validated using an Eastern database, suggesting that it is applicable across races.

The present study has some limitations. First, our study was a retrospective observational study exposed to potential confounding bias; a larger multicenter prospective study may be required. Second, we not only constructed an easyto-use model but also used an Eastern database for the external validation cohort. However, the sample for the external validation cohort was small, and some meaningful metrics, such as carbohydrate antigen 19-9, surgical margin, platelet-to-lymphocyte ratio, and oncogene expression, were lacking. Multidimensional factors pertaining to the tumor, microenvironment, and host should be considered in a good prediction model, as adding these factors may improve the quality of the nomogram. Therefore, a larger multicenter prospective study should be performed to verify the conclusions of our study.

\section{Conclusions}

Distinct clinical characteristics among different individuals lead to different outcomes after curative resection. We established a novel nomogram that combined clinicopathological features, AJCC T stage, and therapy methods, showed satisfactory predictive power in PLN$\mathrm{PaCs}$ following surgical resection, and might be a good model for application in the clinic. Our easy-to-use nomogram could not only define a high-risk population based on the nomo-score but also indicate that adjuvant radiotherapy was a critical prognostic factor in PLN$\mathrm{PaCs}$. Based on these findings, more laboratory indexes and genetic information should be explored in the future to promote individualized treatment for PLN-PaCs.

\section{Acknowledgments}

Funding: This study was supported by the Guangdong Medical Science and Technology Research Fund (A2018128).

\section{Footnote}

Reporting Checklist: The authors have completed the TRIPOD reporting checklist. Available at http://dx.doi. org/10.21037/gs-20-597

Conflicts of Interest: All authors have completed the ICMJE uniform disclosure form (available at http://dx.doi. org/10.21037/gs-20-597). The authors have no conflicts of interest to declare. 
Ethical Statement: The authors are accountable for all aspects of the work in ensuring that questions related to the accuracy or integrity of any part of the work are appropriately investigated and resolved. The study was conducted in accordance with the Declaration of Helsinki (as revised in 2013).

Open Access Statement: This is an Open Access article distributed in accordance with the Creative Commons Attribution-NonCommercial-NoDerivs 4.0 International License (CC BY-NC-ND 4.0), which permits the noncommercial replication and distribution of the article with the strict proviso that no changes or edits are made and the original work is properly cited (including links to both the formal publication through the relevant DOI and the license). See: https://creativecommons.org/licenses/by-nc-nd/4.0/.

\section{References}

1. Huang L, Jansen L, Balavarca Y, et al. Resection of pancreatic cancer in Europe and USA: an international large-scale study highlighting large variations. Gut 2019;68:130-9.

2. Moyer MT GR. Pancreatic adenocarcinoma. N Engl J Med 2014;371:2140.

3. Krempien R, Roeder F. Intraoperative radiation therapy (IORT) in pancreatic cancer. Radiat Oncol 2017;12:8.

4. Zaki Azzam A, Alqarni A, Mahmoud Amin T. The role of intraoperative radiotherapy (IORT) in the management of patients with pancreatic and periampullary cancer: A single center experience. J Egypt Natl Canc Inst 2018;30:77-9.

5. Hidalgo M. Pancreatic cancer. N Engl J Med 2010;362:1605-17.

6. Song X, Shao Z, Liang H. Using the new INTRABEAM mobile intraoperative radiotherapy system during surgery for pancreatic cancer: a case report. J Med Case Rep 2019;13:23.

7. Tempero MA, Malafa MP, Al-Hawary M, et al. Pancreatic Adenocarcinoma, Version 2.2017, NCCN Clinical Practice Guidelines in Oncology. J Natl Compr Canc Netw 2017;15:1028-61.

8. Warschkow R, Tsai C, Köhn N, et al. Role of lymphadenectomy, adjuvant chemotherapy, and treatment at high-volume centers in patients with resected pancreatic cancer-a distinct view on lymph node yield. Langenbecks Arch Surg 2020;405:43-54.

9. Eskander MF, de Geus SW, Kasumova GG, et al. Evolution and impact of lymph node dissection during pancreaticoduodenectomy for pancreatic cancer. Surgery 2017;161:968-76.

10. Kamarajah SK, Burns WR, Frankel TL, et al. Validation of the American Joint Commission on Cancer (AJCC) 8th Edition Staging System for Patients with Pancreatic Adenocarcinoma: A Surveillance, Epidemiology and End Results (SEER) Analysis. Ann Surg Oncol 2017;24:2023-30.

11. Schorn S, Demir IE, Haller B, et al. The influence of neural invasion on survival and tumor recurrence in pancreatic ductal adenocarcinoma - A systematic review and meta-analysis. Surg Oncol 2017;26:105-15.

12. Aziz H, Zeeshan M, Jie T, et al. Neoadjuvant Chemoradiation Therapy Is Associated with Adverse Outcomes in Patients Undergoing Pancreaticoduodenectomy for Pancreatic Cancer. Am Surg 2019;85:1276-80.

13. Loveday BPT, Lipton L, Thomson BN. Pancreatic cancer: An update on diagnosis and management. Aust J Gen Pract 2019;48:826-31.

14. Iasonos A, Schrag D, Raj GV, et al. How to build and interpret a nomogram for cancer prognosis. J Clin Oncol 2008;26:1364-70.

15. Sternberg CN. Are nomograms better than currently available stage groupings for bladder cancer? J Clin Oncol 2006;24:3819-20.

16. Tian T, Zhang P, Zhong F, et al. Nomogram construction for predicting survival of patients with non-small cell lung cancer with malignant pleural or pericardial effusion based on SEER analysis of 10,268 patients. Oncol Lett 2020;19:449-59.

17. Han L, Dai W, Mo S, et al. Nomogram to predict the risk and survival of synchronous bone metastasis in colorectal cancer: a population-based real-world analysis. Int J Colorectal Dis 2020;35:1575-85.

18. Fakhry C, Zhang Q, Nguyen-Tân PF, et al. Development and validation of nomograms predictive of overall and progression-free survival in patients with oropharyngeal cancer. J Clin Oncol 2017;35:4057-65.

19. Lu Z, Yan W, Liang J, et al. Nomogram Based on Systemic Immune-Inflammation Index to Predict Survival of Tongue Cancer Patients Who Underwent Cervical Dissection. Front Oncol 2020;11;10:341.

20. Wolbers M, Koller MT, Witteman JC, et al. Prognostic models with competing risks: methods and application to coronary risk prediction. Epidemiology 2009;20:555-61.

21. Van Calster B, Nieboer D, Vergouwe Y, et al. A calibration hierarchy for risk models was defined: from utopia to empirical data. J Clin Epidemiol 2016;74:167-76. 
22. Van Calster B, Wynants L, Verbeek JFM, et al. Reporting and Interpreting Decision Curve Analysis: A Guide for Investigators. Eur Urol 2018;74:796-804.

23. Ansari D, Althini C, Ohlsson H, et al. Early-onset pancreatic cancer: a population-based study using the SEER registry. Langenbeck's Archives of Surgery 2019;404:565-71.

24. Wu M, Li X, Zhang T, et al. Identification of a Nine-Gene Signature and Establishment of a Prognostic Nomogram Predicting Overall Survival of Pancreatic Cancer. Front Oncol 2019;9:996.

25. He C, Zhang Y, Cai Z, et al. Overall survival and cancerspecific survival in patients with surgically resected pancreatic head adenocarcinoma: A competing risk nomogram analysis. J Cancer 2018;9:3156-67.

26. Okamura Y, Yasukawa S, Narimatsu H, et al. The hENT1 expression is a predictor in patients with resected pancreatic cancer treated with adjuvant S-1 chemotherapy. Cancer Sci 2020;111:548-60.

27. Hlavsa J, Cecka F, Zaruba P, et al. Tumor grade as significant prognostic factor in pancreatic cancer: validation of a novel TNMG staging system. Neoplasma 2018;65:637-43.

28. Warschkow R, Widmann B, Beutner U, et al. The More the Better-Lower Rate of Stage Migration and Better Survival in Patients With Retrieval of 20 or More Regional Lymph Nodes in Pancreatic Cancer: A Population-Based Propensity Score Matched and Trend SEER Analysis. Pancreas 2017;46:648-57.

29. Song W, Miao DL, Chen L. Nomogram for predicting survival in patients with pancreatic cancer. Onco Targets Ther 2018;11:539-45.

30. Slidell MB, Chang DC, Cameron JL, et al. Impact of total lymph node count and lymph node ratio on staging and survival after pancreatectomy for pancreatic adenocarcinoma: a large, population-based analysis. Ann Surg Oncol 2008;15:165-74.

31. Schwarz RE, Smith DD. Extent of lymph node retrieval and pancreatic cancer survival: information from a large

Cite this article as: Jin L, Zou Y, Ruan S, Han H, Zhang Y, Chen Z, Jin H, Shi N. Score for predicting overall survival in pancreatic adenocarcinoma patients with positive lymph nodes after surgery: a novel nomogram-based risk assessment. Gland Surg 2021;10(2):529-540. doi: 10.21037/gs-20-597
US population database. Ann Surg Oncol 2006;13:1189200.

32. Tomlinson JS JS, Bentrem DJ, et al. Accuracy of staging node-negative pancreas cancer: a potential quality measure. Arch Surg 2007;142:767-723; discussion 773-4.

33. Showalter TN, Winter KA, Berger AC, et al. The influence of total nodes examined, number of positive nodes, and lymph node ratio on survival after surgical resection and adjuvant chemoradiation for pancreatic cancer: a secondary analysis of RTOG 9704. Int J Radiat Oncol Biol Phys 2011;81:1328-35.

34. Pawlik TM, Gleisner AL, Cameron JL, et al. Prognostic relevance of lymph node ratio following pancreaticoduodenectomy for pancreatic cancer. Surgery 2007;141:610-8.

35. Sierzega M, Popiela T, Kulig J, et al. The Ratio of Metastatic Resected Lymph Nodes is an Independent Prognostic Factor in Patients With Node-positive Pancreatic Head Cancer. Pancreas 2006;33:240-5.

36. Riediger H, Keck T, Wellner U, et al. The lymph node ratio is the strongest prognostic factor after resection of pancreatic cancer. J Gastrointest Surg 2009;13:1337-44.

37. Ashfaq A, Pockaj BA, Gray RJ, et al. Nodal counts and lymph node ratio impact survival after distal pancreatectomy for pancreatic adenocarcinoma. J Gastrointest Surg 2014;18:1929-35.

38. Strobel O, Neoptolemos J, Jager D, et al. Optimizing the outcomes of pancreatic cancer surgery. Nat Rev Clin Oncol 2019;16:11-26.

39. Oettle H, Neuhaus P, Hochhaus A, et al. Adjuvant chemotherapy with gemcitabine and long-term outcomes among patients with resected pancreatic cancer: the CONKO-001 randomized trial. Jama 2013;310:1473-81.

40. Maeda A, Boku N, Fukutomi A, et al. Randomized phase III trial of adjuvant chemotherapy with gemcitabine versus S-1 in patients with resected pancreatic cancer: Japan Adjuvant Study Group of Pancreatic Cancer (JASPAC-01). Jpn J Clin Oncol 2008;38:227-9. 
Supplementary

Table S1 Detailed scores of all variables in the nomogram.

\begin{tabular}{|c|c|}
\hline Variables & Nomogram score for OS \\
\hline \multicolumn{2}{|l|}{ Grade } \\
\hline Well & 0 \\
\hline Moderately & 58 \\
\hline Poorly & 90 \\
\hline \multicolumn{2}{|l|}{ Radiotherapy } \\
\hline No & 23 \\
\hline Yes & 0 \\
\hline \multicolumn{2}{|c|}{ Chemotherapy } \\
\hline No & 92 \\
\hline Yes & 0 \\
\hline \multicolumn{2}{|l|}{ LNR } \\
\hline$\leq 0.12$ & 0 \\
\hline $0.12-0.38$ & 32 \\
\hline$\geq 0.38$ & 81 \\
\hline \multicolumn{2}{|l|}{ AJCC T.stage } \\
\hline $\mathrm{T} 1$ & 0 \\
\hline $\mathrm{T} 2$ & 38 \\
\hline T3 & 71 \\
\hline T4 & 100 \\
\hline \multicolumn{2}{|l|}{ Age } \\
\hline$<50$ & 0 \\
\hline 70 & 18 \\
\hline$\geq 70$ & 54 \\
\hline
\end{tabular}

OS, overall survival, AJCC, American Joint Committee for Cancer; LNR, lymph nodes ratio. 